OPEN ACCESS

Edited by:

Sang-Bae Ko,

Seoul National University Hospital,

South Korea

Reviewed by:

Chia-Yi (Alex) Kuan,

Emory University, United States

Marco Matteo Ciccone,

University of Bari Aldo Moro, Italy

${ }^{*}$ Correspondence:

Mona N. Bahouth mbahout1@jhmi.edu

Specialty section:

This article was submitted to

Stroke,

a section of the journa

Frontiers in Neurology

Received: 28 August 2021 Accepted: 11 January 2022 Published: 08 March 2022

Citation:

Bahouth MN, Saylor D, Hillis AE and Gottesman RF (2022) The Impact of Mean Arterial Pressure and Volume Contraction in With Acute Ischemic Stroke. Front. Neurol. 13:766305. doi: 10.3389/fneur.2022.766305

\section{The Impact of Mean Arterial Pressure and Volume Contraction in With Acute Ischemic Stroke}

\author{
Mona N. Bahouth ${ }^{1,2 *}$, Deanna Saylor ${ }^{1}$, Argye E. Hillis ${ }^{1}$ and Rebecca F. Gottesman ${ }^{1,3}$ \\ ${ }^{1}$ School of Medicine, Johns Hopkins University, Baltimore, MD, United States, ${ }^{2}$ School of Nursing, Johns Hopkins University, \\ Baltimore, MD, United States, ${ }^{3}$ School of Public Health, Johns Hopkins University, Baltimore, MD, United States
}

Background and Purpose: Hydration at the time of stroke may impact functional outcomes. We sought to investigate the relationship between blood pressure, hydration status, and stroke severity in patients with acute ischemic stroke (AIS).

Methods: We evaluated hydration status, determined by blood urea nitrogen

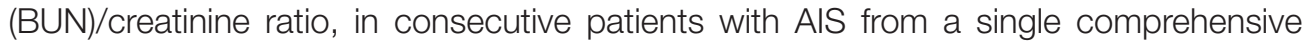
stroke center. Baseline mean arterial pressure (MAP) was analyzed using a linear spline with a knot at $90 \mathrm{mmHg}$. Baseline stroke severity was defined based on admission $\mathrm{NIH}$ Stroke Scale scores (NIHSSS) and MRI diffusion-weighted imaging.

Results: Among 108 eligible subjects, 55 (51\%) presented in a volume contracted state. In adjusted models, in the total sample, for every $10 \mathrm{mmHg}$ higher MAP up to 90 mmHg, NIHSSS was 2.8 points lower $(p=0.053)$, without further statistically significant association between MAP above 90 and NIHSSS. This relationship was entirely driven by the individuals in a volume contracted state: MAP was not associated with NIHSSS in individuals who were euvolemic. For individuals in a volume contracted state, each $10 \mathrm{mmHg}$ higher MAP, up to $90 \mathrm{mmHg}$, was associated with 6.9 points lower NIHSSS (95\% $\mathrm{Cl}-11.1,-2.6)$. MAP values above $90 \mathrm{mmHg}$ were not related to NIHSSS in either dehydrated or euvolemic patients.

Conclusions: Lower MAP contributes to more severe stroke in patients who are volume contracted, but not those who are euvolemic, suggesting that hydration status and blood pressure may jointly contribute to the outcome. Hydration status should be considered when setting blood pressure goals for patients with AIS.

Keywords: hydration, blood pressure, stroke severity, acute ischemic stroke, hospital care, early recovery

\section{INTRODUCTION}

Despite advances in stroke care and identification of acute therapies for ischemic stroke, stroke remains a leading cause of adult disability. (1). Blood pressure management is a critical component of patient management in the early stroke recovery period. A growing body of evidence suggests that patients who are dehydrated, volume contracted, or both at the time of stroke have worse functional outcomes independent of age, size of the stroke, or presence of complex comorbidities $(2,3)$. The mechanism behind this relationship is yet unknown, though many hypothesize that the worse outcome is due to blood pressure variations that alter cerebral perfusion during a period of disrupted autoregulation. We propose that hydration status may play an independent role. 
Surprisingly, rehydration after stroke has received little attention in the United States outside of a small study of induced hypertension and a series of hemodilution studies (4-7). Expert consensus drives the current acute stroke recommendation for rehydration for patients who are in a volume contracted state (8). These are challenging to implement as there is no single objective measure for dehydration and the duration of therapy is not specified especially in the setting of comorbid conditions (9). There are several approaches to quantifying hydration status with varying levels of data to support such practices: noninvasive cardiac output monitoring with passive leg raise and fluid challenge, bioelectric impedance vector analysis, and serum markers of hydration status (10-14). In this study, we use blood urea nitrogen (BUN) to creatinine ratio as a surrogate objective marker of hydration status with the threshold for volume contracted state as BUN/creatinine $>15$ since it is readily available for use globally (15-17). We sought to explore the relationship between hydration status, blood pressure, and stroke severity in order to test the relative contributions of these physiologic factors in patients with acute ischemic stroke (AIS). Specifically, the present study sought to determine whether or not hydration status modifies the relationship between blood pressure and stroke severity. We additionally wished to identify similar relationships between hydration and blood pressure and longer-term functional outcome, measured at 3 months using modified Rankin Scores (mRS).

\section{METHODS}

\section{Data Source}

Consecutive patients with ischemic stroke were prospectively screened for eligibility between 2014 and 2015. Subjects were included if they had MRI-confirmed ischemic stroke within 12$h$ from stroke onset and did not have signs of active infection, gastrointestinal bleeding, or chronic kidney disease. Indirect measures of volume status including BUN/creatinine ratio were collected with a threshold of BUN/creatinine ratio $>15$ sets as the indicator of a volume contracted state. Stroke severity was determined by the NIH Stroke Scale (NIHSSS) at the time of hospital presentation. Mean arterial pressure (MAP) was calculated using standard equations based on the first blood pressure obtained on arrival to the hospital. Infarct volume was calculated using baseline MRI diffusion-weighted imaging (DWI) sequences and diffusion to perfusion (PWI) mismatch calculated if perfusion study was obtained as the standard of care upon admission to the hospital using OleaSphere software (OleaSphere 3; Olea Medical, La Ciotat, France) by a single primary rater blinded to both diagnosis and hydration status. The study was approved by the university's Institutional Review Board.

\section{Statistical Analysis}

Statistical analysis was performed using Stata Statistical software version 13 (StataCorp LP, College Station, TX, USA). The primary outcome was stroke severity defined by NIHSSS. Comparisons of key clinical characteristics based on hydration status and MAP were made using Fisher's exact tests for categorical variables and the $t$-tests for continuous variables. Given the appearance during descriptive analysis using a LOWESS curve of a non-linear pattern between MAP and NIHSSS, a linear spline was used, with a single knot at $90 \mathrm{mmHg}$.

Statistically significant variables derived from univariable analysis as well as other variables that may be plausibly associated with stroke severity were considered as covariates; however, final model selection was based on the Akaike Information Criterion. Multivariable linear regression models evaluating MAP using this spline, and considering NIHSSS as the dependent variable, were adjusted for age, sex, atrial fibrillation, diabetes, and infarct volume. Exploratory analysis of the functional outcome was based on an a priori dichotomized mRS: $0-1=$ favorable functional outcome and $>1=$ poor outcome, in similarlyadjusted logistic regression models.

To investigate the relationship between hydration status and blood pressure at the time of stroke, we used the above-described linear regression models, adjusted for the same potential confounders, evaluating the effect of MAP on baseline NIHSSS, but stratified models by hydration status, along with formal testing for interaction between MAP and hydration status. Univariate analysis and multivariable logistic regression models were used to evaluate dichotomized 3month outcomes (mRS $0-1$ or $\mathrm{mRS}>1$ ) by hydration status and MAP.

\section{RESULTS}

A total of 312 subjects were screened for this study. Among those 185 subjects were excluded for reasons of presentation to the hospital $>12 \mathrm{~h}$ from stroke onset, unable to complete MRI or presence of baseline renal disease. Of the 126 subjects remaining, 108 had sufficient labs and baseline blood pressure data for analysis of which $55(51 \%)$ were in a volume contracted state at the time of hospital presentation. Mean initial MAPs in the volume contracted and euvolemic groups were $112.3 \mathrm{mmHg}$ and $112.5 \mathrm{mmHg}$ respectively $(p=0.97)$. With this sample size, we have $80 \%$ power to detect a difference between groups with alpha 0.05 (Table 1).

In adjusted models including the total sample, for every $10 \mathrm{mmHg}$ higher MAP up to $90 \mathrm{mmHg}$, NIHSSS was 2.8 points lower $(p=0.053)$, without further statistically significant association between MAP above $90 \mathrm{mmHg}$ and NIHSSS (Table 2). When analyses were stratified, however, it was apparent that this relationship was entirely driven by the individuals in a volume contracted state: MAP was not associated with NIHSSS in individuals who were euvolemic. For individuals in a volume contracted state, each $10 \mathrm{mmHg}$ higher MAP, up to $90 \mathrm{mmHg}$, was associated with 6.9 points lo8wer NIHSSS (95\% CI $-11.1,-2.6 ; p=0.002)$ (Figure 1). The formal test for interaction between MAP and dehydration status for MAP values below $90 \mathrm{mmHg}$ was statistically significant $(p=0.01)$. For subjects with initial MAP above $90 \mathrm{mmHg}$, there was no association between increased MAP and severity in the volume contracted or euvolemic groups. There was 
TABLE 1 | Demographics for the analytic sample.

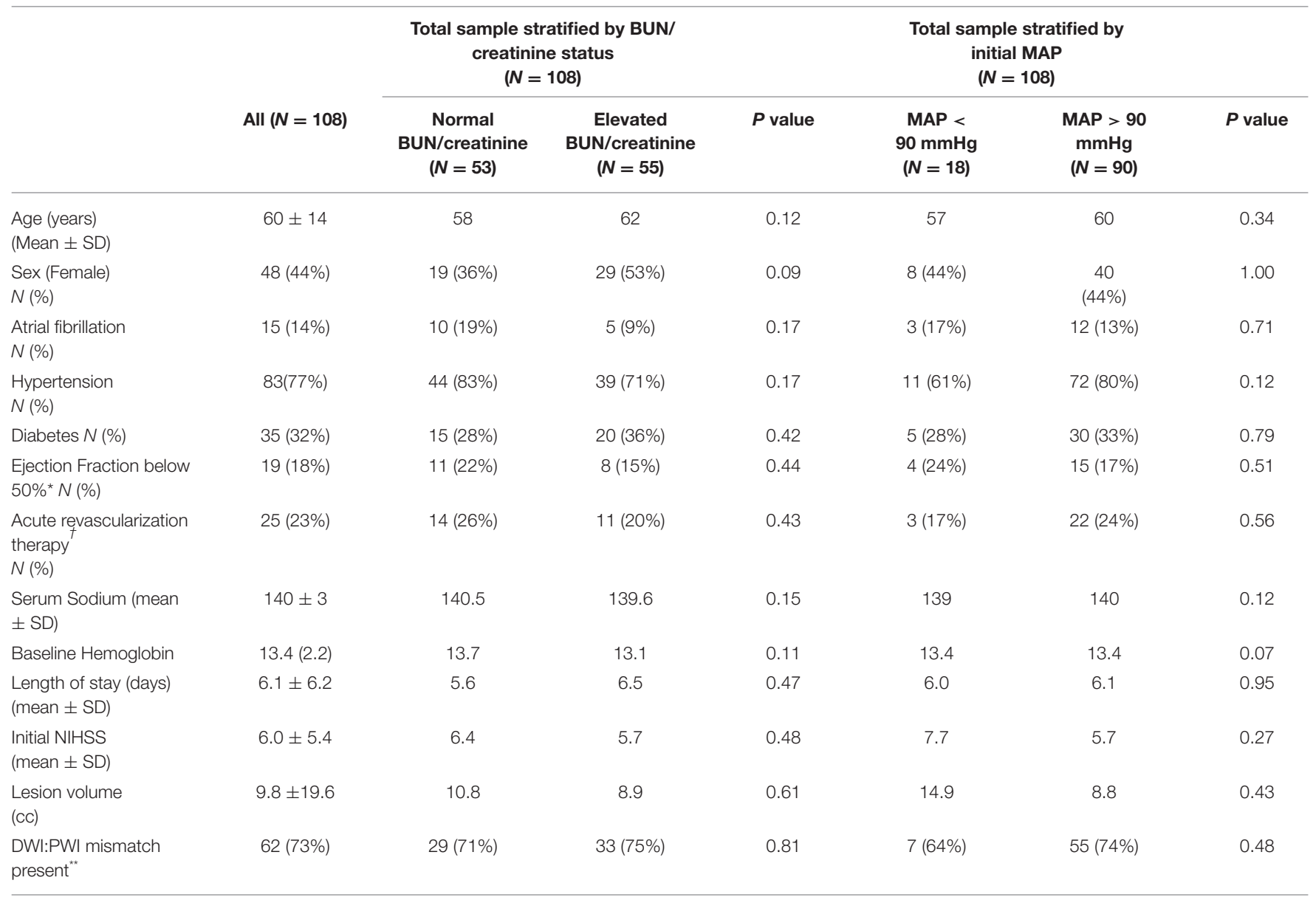

"Ejection Fraction available on $N=103 .{ }^{\dagger}$ (Intravenous tPA and/or endovascular therapy). Lesion volume available on N=108; " DWI:PWI mismatch defined as > 1.2; N=85.

TABLE 2 | Effect of mean arterial pressure (per $10 \mathrm{~mm} \mathrm{Hg}$ ) on stroke severity and outcome: multivariable regression analysis.

\begin{tabular}{|c|c|c|c|c|c|c|}
\hline & \multicolumn{3}{|c|}{$\begin{array}{l}\text { Stroke severity: baseline NIHSS } \\
\text { score }(N=108)\end{array}$} & \multicolumn{3}{|c|}{$\begin{array}{l}\text { Functional outcome: } 3 \text { month } \\
\text { modified rankin scale (mRS }>1 \text { vs. } \\
\text { mRS } 0-1)^{\star *}\end{array}$} \\
\hline All patients & 108 & $-2.8(-5.6,0.04)$ & $-0.1(-0.3,0.5)$ & 86 & $0.25(0.02,3.42)$ & $1.11(0.86,1.44)$ \\
\hline Euvolemic patients & 53 & $1.3(-3.0,5.5)$ & $-0.1(-0.7,0.4)$ & 41 & $0.25(0.007,8.93)$ & $0.98(0.69,1.39)$ \\
\hline
\end{tabular}

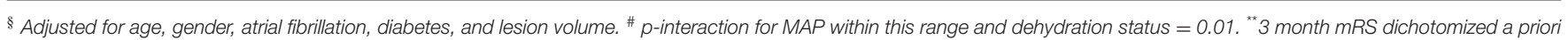
as bad outcome ( $m R S>1)$ vs. good outcome (mRS 0-1).

no difference in odds of poor 3-month outcome mRS based on differences in initial MAP (unadjusted OR 1.05; 95\% CI $0.85,1.29)$.

In terms of MRI findings, the size of the stroke lesion was measurable in all 108 subjects. The average ischemic lesion size based on diffusion-weighted imaging was $9.8 \pm 19.6 \mathrm{cc}$. In this cohort, 84/108 (78\%) subjects had perfusion imaging sufficient for interpretation if diffusion to perfusion mismatch. There was no difference in diffusion to perfusion mismatch based on hydration status nor mean arterial pressure. 


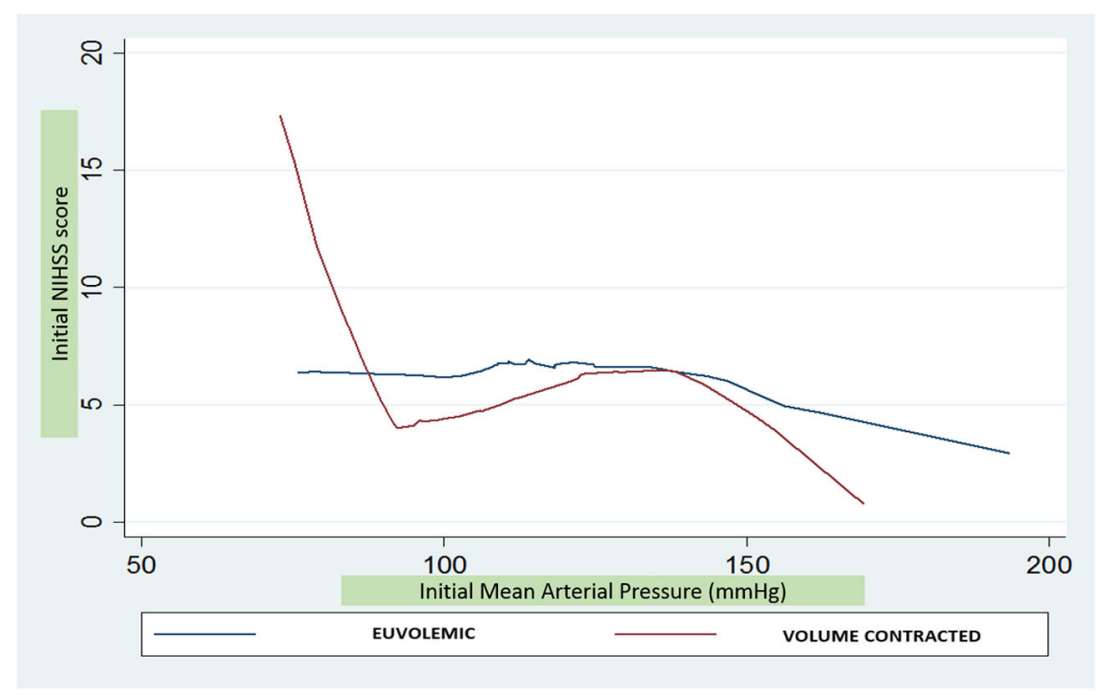

FIGURE 1 | Comparison of NIH stroke scale score and mean arterial pressure by hydration status.

\section{DISCUSSION}

Although limited by a relatively small sample size, these data suggest that lower MAP may be an important variable in subjects who are volume contracted at the time of stroke. It suggests that volume contracted patients with ischemic stroke may be at higher risk for more severe stroke, particularly if they also have low blood pressure. Notably, there was no significant difference between blood pressures in the volume contracted and euvolemic patients.

A large percentage of patients with AIS are volume contracted at the time of stroke $(2-4,15,16)$. Intravascular volume is one component in blood pressure and avoidance of low blood pressure has been standard in acute stroke care (8). These data suggest that avoidance of low blood pressure may be especially important in patients who are dehydrated. Therefore, hydration status may be an important consideration when setting early blood pressure goals.

This study also expands on the knowledge generated from earlier studies of induced hypertension as a potentially helpful adjunct therapy after ischemic stroke (18-20). These studies used a combination of pharmacological agents and volume expanders to elevate blood pressure at the time of stroke and demonstrated improved clinical outcomes. Our results suggest an opportunity for potential benefit using a simpler treatment using solely intravenous saline. This is especially important given that the largest burden of stroke worldwide is in lowresourced settings, and intravenous saline could represent a scalable intervention to improve stroke outcomes even in these settings.

There are multiple potential reasons for hydration and stroke severity to be linked, independent of MAP. First, blood viscosity may be impacted by hydration, such that lower viscosity improves perfusion. Next, patients who are chronically volume contracted may have more accumulation of brain disease prior to the stroke event thus impacting severity. Finally, comorbidities such as diabetes and heart failure might be associated with volume contraction and influence outcome. This study further clarifies the relationship between hydration status, blood pressure, and stroke severity. That is, the combination of low blood pressure and volume contraction is more strongly associated with higher stroke severity than either alone.

This study has several limitations, including those common to the small sample size. Further, only $80 \%$ of these subjects had 3 month mRS completed limiting our ability to assess functional outcomes in the entire cohort. Next, data collected within the standard care environment are subject to variation in technique, specifically when related to the measurement of initial blood pressure. Nevertheless, these data yield the important finding that blood pressure and hydration status are potentially independent factors contributing to stroke severity, independent of infarct volume, and suggest that dehydrated stroke patients with lower MAP are at high risk. This high-risk subgroup may benefit from rehydration strategies.

\section{CONCLUSIONS}

Lower MAP contributes to more severe stroke in patients who are volume contracted but not in those who are euvolemic. The topic of patient hydration status may deserve more attention in the early treatment period after stroke. These results suggest a potentially modifiable risk factor to improve functional outcomes with low cost, broadly available interventions like rehydration. 


\section{DATA AVAILABILITY STATEMENT}

Raw data will be made available for any reasonable request of the authors.

\section{ETHICS STATEMENT}

The studies involving human participants were reviewed and approved by Institutional Review Board, Johns Hopkins School of Medicine. Written informed consent for participation was not required for this study in accordance with the national legislation and the institutional requirements.

\section{REFERENCES}

1. Virani SS, Alonso A, Benjamin EJ, Bittencourt MS, Callaway CW, Carson SP, et al. Heart Disease and stroke statistics -2020. Circulation. (2020) 141:139596. doi: 10.1161/CIR.0000000000000757

2. Schrock JW, Glasenapp M, Drogell K. Elevated blood urea nitrogen/creatinine ratio is associated with poor outcome in patients with ischemic stroke. Clin Neurol Neurosurg. (2012) 114:881-4. doi: 10.1016/j.clineuro.2012.01.031

3. Lin LC, Yang JT, Weng HH, Hsaio CT, Lai SL, Fann WC. Predictors of early clinical deterioration after ischemic stroke. Am J Emerg Med. (2011) 29:577-81. doi: 10.1016/j.ajem.2009.12.019

4. Bhalla A, Sankaralingam S, Dundas R, Swaminathan R, Wolfe CD, Rudd AG. Influence of raised plasma osmolality on clinical outcome after acute stroke. Stroke. (2000) 31:2043-8. doi: 10.1161/01.STR.31.9.2043

5. Bath PMW, Krishnan K. Interventions for deliberately altering blood pressure in acute stroke. Cochr Datab System Rev. (2014) 10:CD000039. doi: 10.1002/14651858.CD000039.pub3

6. Chalela JA, Dun B, Todd JW, Warach S. Induced hypertension improves cerebral blood flow in acute ischemic stroke. Neurology. (2005) 64:1979. doi: 10.1212/01.WNL.0000156360.70336.18

7. Chang TS, Jensen MB. Haemodilution for acute ishaemic stroke. Cochr Datab System Rev. (2014) 10:CD000103. doi: 10.1002/14651858.CD000103.pub2

8. Jauch EC, Saver JL, Adams HP, Bruno A, Connors JJ, Demaerschalk BM, et al. Guidelines of the early management of patients with acute ischemic stroke. Stroke. (2013) 44:870-947. doi: 10.1161/STR.0b013e318284056a

9. Armstrong LE. Assessing hydration status: the elusive gold standard. J Am Coll Nutr. (2007) 26:575S-584S. doi: 10.1080/07315724.2007.10719661

10. Marik PE. Noninvasive cardiac output monitors: a state-of -the-art review. J Cardiothor Vasc Anesth. (2013) 27:121-34. doi: 10.1053/j.jvca.2012.03.022

11. Squara P, Denjean D, Estagnasie P, Brusset A, Dib JC, Dubois C. Noninvasive cardiac output monitoring (NICOM): a clinical validation. Intensive Care Med. (2007) 22:1191-4. doi: 10.1007/s00134-007-0640-0

12. Squara P, Rotcajg D, Denjean D, Estagnasie P, Brusset A. Comparison of monitoring performance of bioreactance versus pulse contour during lung recruitment maneuvers. Crit Care. (2009) 13:1-6. doi: 10.1186/cc7981

13. Cavallaro F, Sandroni C, Marano C, La Torre G, Mannocci A, De Waure C, et al. Diagnostic accuracy of passive leg raising for prediction of fluid responsiveness in adults: systematic review and meta-analysis of clinical studies. Intensive Care Med. (2010) 36:1475-83. doi: 10.1007/s00134-010-1929-y

14. Scicchitano P, Massari F. Bioimpedence vector analysis in the evaluation of congestions in heart failure. Biomark Med. (2020) 14:2019. doi: 10.2217/bmm-2019-0429

\section{AUTHOR CONTRIBUTIONS}

All authors listed have made a substantial, direct, and intellectual contribution to the work and approved it for publication.

\section{FUNDING}

This work was supported by NIH (NINDS) Grants R01NS047691 to AH, R25 NS065729 to AH with supplement to MB; Richard Starr Ross Clinician Scientist Association Grant to MB, K24 AG052573 to RG, and the American Heart Association Career Development Grant to MB.

15. Bhatia K, Mohanty S, Tripathi BK, Gupta B, Mittal MK. Predictors of early neurological deterioration in patients with acute ischaemic stroke with special reference to $\mathrm{BUN} /$ creatinine ratio \& urine specific gravity. Indian J Med Res. (2015) 141:299-307. doi: 10.4103/0971-5916.1 56564

16. Bahouth MN, Bahrainwala Z, Hillis AE, Gottesman RF. Dehydration status is associated with more hemispatial neglect after stroke. Neurologist. (2016) 21:101-5. doi: 10.1097/NRL.00000000000 00101

17. Liu CH, Lin SC, Lin JR, Yang JT, Chang YJ, Chang CH, et al. Dehydration is an independent predictor of discharge outcome and admission cost in acute ischaemic stroke. Eur J Neurol. (2014) 21:1184-91. doi: 10.1111/ene. 12452

18. Rordorf G, Koroshetz WJ, Ezzeddine MD, Segal AZ, Buonanno FS. A pilot study of drug-induced hypertension for treatment of acute stroke. Neurology. (2001) 56:1210-3. doi: 10.1212/WNL.56. 9.1210

19. Hillis AE, Ulatowski JA, Barker PB, Terbey M, Ziai W, Beauchamp NJ, et al. A pilot randomized trial of induced blood pressure elevation. Cerebrov Dis. (2003) 16:236-46. doi: 10.1159/0000 71122

20. Koenig MA, Geocadin RG, deGrouchy M, Glasgow J, Vimal S, Restrepo L, et al. Safety and induced hypertension therapy in patients with acute ischemic stroke. Neurocritical Care. (2006) 4:3-7. doi: 10.1385/NCC:4:1:003

Conflict of Interest: The authors declare that the research was conducted in the absence of any commercial or financial relationships that could be construed as a potential conflict of interest.

Publisher's Note: All claims expressed in this article are solely those of the authors and do not necessarily represent those of their affiliated organizations, or those of the publisher, the editors and the reviewers. Any product that may be evaluated in this article, or claim that may be made by its manufacturer, is not guaranteed or endorsed by the publisher.

Copyright (C) 2022 Bahouth, Saylor, Hillis and Gottesman. This is an open-access article distributed under the terms of the Creative Commons Attribution License (CC $B Y)$. The use, distribution or reproduction in other forums is permitted, provided the original author(s) and the copyright owner(s) are credited and that the original publication in this journal is cited, in accordance with accepted academic practice. No use, distribution or reproduction is permitted which does not comply with these terms. 